\title{
Impact of Type II Diabetes on the Presentation of Coronary Artery Disease and Outcomes of Percutaneous Coronary Intervention in Female Patients
}

\author{
Rama Kishore Yalampati ${ }^{1}$ Anne M. Beatrice ${ }^{2}$ \\ ${ }^{1}$ Department of Cardiology, Nizam's Institute of Medical Sciences, \\ Punjagutta, Hyderabad, Telangana, India \\ 2Department of Endocrinology, Nizam's Institute of Medical \\ Sciences, Punjagutta, Hyderabad, Telangana, India \\ Indian J Cardiovasc Dis Women-WINCARS 2018;3:12-16
}

Address for correspondence Rama Kishore Yalampati, DM Student, Department of Cardiology, Nizam's Institute of Medical Sciences, Punjagutta, Hyderabad 500082, Telangana, India (e-mail: honeyyrk@gmail.com).

\section{Abstract}

Background Coronary artery disease (CAD) is a well-established complication of type II diabetes mellitus affecting both men and women worldwide. A vast amount of research has shown evidence of increased risk for CAD in patients with type II diabetes compared with the general population and that this increased relative risk is more prevalent in women. The primary aim of this study is to observe the impact of type II diabetes on the severity of CAD at the time of presentation and also on the outcomes of $\mathrm{CAD}$ after $\mathrm{PCl}$ (percutaneous coronary intervention) in female patients.

Methods A prospective single-center study for the period of 1 year, including 267 female CAD patients who underwent $\mathrm{PCl}$ was conducted. The patient population was divided into two major groups of which 171 (64\%) patients had type II diabetes (group A) and 96 (36\%) patients were nondiabetic (group B). The clinical presentations of CAD in type II diabetics and nondiabetics were compared in the form of chronic stable angina (CSA), acute coronary syndrome (ACS), left ventricular (LV) dysfunction, renal dysfunction, and the number of vessels involved. Primary outcomes were compared in the form of major adverse cardiovascular events (MACE), whereas secondary outcomes were compared in the form of statin-induced myopathy, also referred to as contrast-induced nephropathy (CIN).

Results At presentation, 60 (35.08\%) of 171 female patients with type II diabetes (group A) had CSA and 121 (70.76\%) had ACS. Baseline creatinine was determined to be $1.17 \pm 1.02 \mathrm{mg} / \mathrm{dL}$ amongst the participants. Moreover, 51 (29.8\%) patients were observed to have LV dysfunction. The total number of lesions treated in this group was 237. Among group B, that is, 96 nondiabetic female patients, 62 (64.5\%) patients had CSA, $34(35.4 \%)$ had ACS, 39 (40.6\%) had LV dysfunction, the baseline creatinine was $(0.95 \pm 0.39) \mathrm{mg} / \mathrm{dL}$. The total number of lesions treated was in this group was 130.

The difference in incidence of CSA, ACS, and renal dysfunction was statistically significant with $p=0.000,0.000$, and 0.016 , respectively. The incidence of CSA was greater in group $B$, that is, nondiabetics; whereas, the incidence of ACS and renal dysfunction was found to be greater in group A, that is, type II diabetics. Moreover, the 


\section{Keywords}

- type II diabetes mellitus

- coronary artery disease

- percutaneous coronary intervention number of vascular territories involved was found to be more in patients with type II diabetes. Although, the incidence of severe LV dysfunction was greater in patients with type II diabetes, the difference was not statistically significant.

When considering 1 year outcomes in female patients with type II diabetes, three cases of deaths, one case of acute stent occlusion, one subacute stent occlusion, one peri-procedural myocardial infarction (MI), one CSA, one statin-induce myopathy, and one rhabdomyolysis are reported. Whereas, in nondiabetic female patients, one case of death, one CSA, one CIN, one chronic thrombus right radial artery, and one pseudoaneurysm and rupture are reported.

Conclusion The results from this study suggest that in female patients with CAD, ACS presentation is more common in type II diabetes; whereas, CSA presentation is more common in nondiabetics. Moreover, multi-vessel involvement is more common in type II diabetes. Following $\mathrm{PCl}$, there has been an increase in the prevalence of MACE, statin-induced myopathy and rhabdomyolysis in patients with type II diabetes. In conclusion, type II diabetes has been reported to significantly influence the severity of presentation, number of lesions involved, and complications following $\mathrm{PCl}$.

\section{Introduction}

The prevalence of type II diabetes mellitus (DM) is increasing globally making it a public health concern. Moreover, $\mathrm{DM}$ is an established risk factor for cardiovascular disease (CVD) with differential effects on women in comparison with men. ${ }^{1}$ CVD has also been identified as the leading cause of mortality amongst women of all ages. ${ }^{2-4}$ Type II DM is an important predictor of coronary artery disease (CAD) risk and prognosis in women and men.- ${ }^{5-9}$ The increase in CAD risk is higher in female diabetic patients with a relative risk for fatal CAD being 3.5 in women compared with 2.1 in men. ${ }^{8}$ Significant differences with respect to epidemiology, diagnosis, response to therapy, and prognosis of congestive heart failure (CHD) exist between women and men. Hence, these factors should be considered when treating women with known or suspected disease. In addition, women are generally underrepresented in cardiovascular randomized studies, and data suggest that men are referred more commonly than women for appropriate therapeutic procedures in clinical practice. ${ }^{10,11}$ The rate of therapeutic success for CAD is equal in both sexes. However, the complication rates differ, which in turn results in sex-specific profile of benefit. Literature has reported that it is the confounding risk factors (e.g., older age, more diabetes, more hypertension [HTN]) and not the gender itself, which is the cause of increased rates of negative outcomes in women with therapies, such as an early invasive strategy in non-ST-elevation acute coronary syndromes (ACS), and fibrinolysis therapy or primary percutaneous coronary intervention (PCI) in ST-elevation myocardial infarction (MI). ${ }^{12-16}$

Our study was an attempt to observe the impact of type II diabetes on the severity of CAD at the time of presentation and also on the outcomes of CAD after PCI in Indian women with type II DM.

\section{Materials and Methods}

This prospective single-center study included female patients, who were aged between 18 and 75 years, with CAD compromising both ACS (acute coronary syndrome) and CSA (chronic stable angina) who underwent percutaneous coronary intervention (PCI) in our institute from 2009 to 2016. After obtaining ethical clearance from the institutional ethics committee, total 267 cases ( $n=267$ ) were enrolled in this study. Informed consent of patients was obtained before enrolment. Patients were divided into two groups: patients with type II DM (group A) and those with no diabetes (group B). There were 171 (64\%) patients in group A and 96 (36\%) in group B.

In this study, we compared both groups in the presentation of CAD (CSA vs. ACS), the presence of left ventricular (LV) and renal dysfunctions, and the number of coronary vessels involved during coronary angiography.

Primary outcomes were compared in the form of major adverse cardiovascular events (MACE), which are objective measures of acute and/or adverse cardiovascular events (e.g., acute MI, ischemic stroke, coronary arterial occlusion, death), which have been used to assess the effects of various interventions (e.g., rotablation, angioplasty, stenting) or therapeutics (e.g., anti-arrhythmic drugs, statins, angiotensin-converting enzyme $[\mathrm{ACE}]$ inhibitors) in the context of a clinical trial. ${ }^{16}$

Secondary outcomes were compared in the form of statin-induced myopathy and contrast-induced nephropathy (CIN) (National Lipid Association 2006 specifically defined it as complaints of myalgia [muscle pain or soreness], weakness, and/or cramps plus elevation in serum creatinine kinase [CK] $>10 \times$ upper limit of normal [ULN]). CIN has been defined as an absolute increase in serum creatinine of $0.5 \mathrm{mg} / \mathrm{dL}$ or a relative $25 \%$ increase from the baseline value, assessed 48 to 72 hours following intravascular administration of contrast medium. ${ }^{17}$ 
Correlations of blood sugar at presentation and the incidence of MACE in group A were also observed.

The two-tailed Student's $t$-test was used for comparison between the groups. Statistical analysis was done with Minitab version 17 (Minitab, Ltd.). Finally the difference in above-mentioned modalities between both the groups was observed for statistical significance $(p<0.05$ was taken as significant).

\section{Results}

In our study, the mean age of presentation of CAD among patients with type II DM was ( $42 \pm 2.5$ years), whereas for those with no diabetes was ( $51 \pm 3$ years), suggesting that patients with type II DM had an earlier age of presentation.

At the time of presentation among group A, that is, 171 female patients with type II DM, 60 (35.08\%) patients had CSA, 121 (70.76\%) had ACS, and 51 (29.8\%) patients had LV dysfunction. The total number of lesions treated was 237.

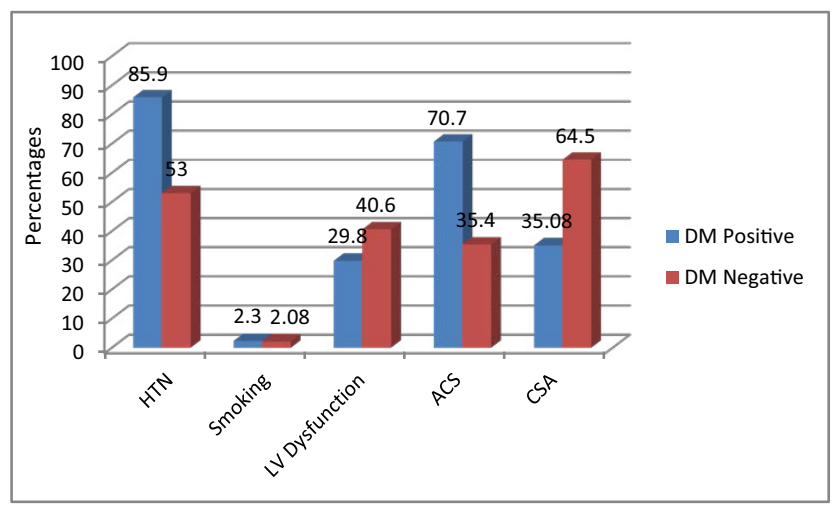

Fig. 1 Comparison of presentation of CAD in diabetes mellitus (DM) versus non-DM. ACS, acute coronary syndrome; CSA, chronic stable angina; HTN, hypertension; LV, left ventricular.
The baseline creatinine at the time of presentation was $1.17 \pm 1.02 \mathrm{mg} / \mathrm{dL}$ in this group (-Fig. 1).

In group $B$, that is, 96 female patients with no diabetes, $62(64.5 \%)$ patients had CSA, 34 (35.4\%) had ACS, and 39 (40.6\%) had LV dysfunction (-Fig. 1). The total number of lesions treated was 130 . The baseline creatinine at the time of presentation was $0.95 \pm 0.39 \mathrm{mg} / \mathrm{dL}$. The differences in the incidence of CSA, ACS, and renal dysfunction between groups A and B were statistically significant with $p=0.000,0.000$, and 0.016 , respectively, suggesting that the incidence of CSA is greater in group B, whereas the incidence of ACS and renal dysfunction at the time of presentation and the number of coronary vascular territories involved were more in group A. Though the incidence of severe LV dysfunction is greater in patients with type II diabetes, the difference was not statistically significant (-Table $\mathbf{1}$ ). The difference in the number of the coronary vessels involved and treated was compared, and the number of the vessels involved was found to be more in type II DM ( - Fig. 2).

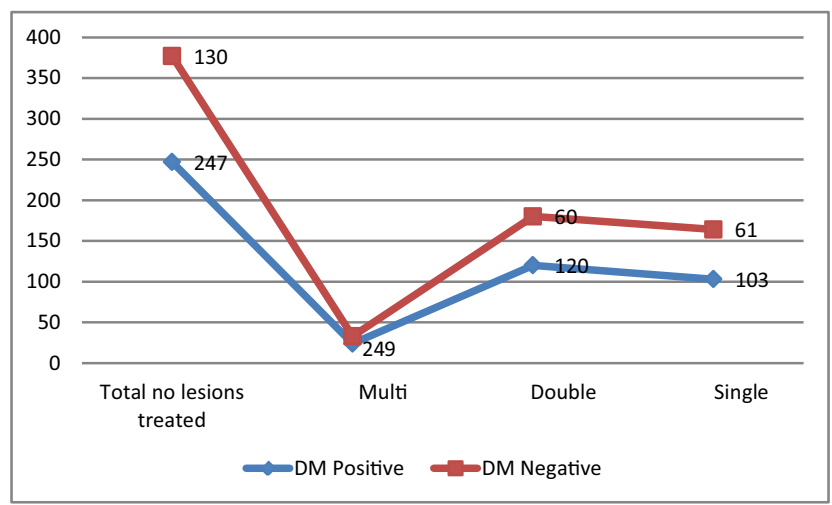

Fig. 2 Comparison of number of vessels treated in diabetics versus nondiabetics. DM, diabetes mellitus.

Table 1 Comparison of type of presentation in diabetics versus nondiabetics and its significance

\begin{tabular}{|l|l|l|l|}
\hline Variable & DM positive & DM negative & $p$ Value \\
\hline Total number & 171 & 96 & \\
\hline HTN & $147(85.9 \%)$ & $51(53.0 \%)$ & 0.000 \\
\hline Smoking & $4(2.3 \%)$ & $2(2.08 \%)$ & 0.891 \\
\hline LV dysfunction & $51(29.8 \%)$ & $39(40.6 \%)$ & 0.077 \\
\hline ACS & $121(70.7 \%)$ & $34(35.4 \%)$ & 0.000 \\
\hline CSA & $60(35.08 \%)$ & $62(64.5 \%)$ & 0.000 \\
\hline Blood urea & $31.74 \pm 18.61$ & $31.66 \pm 16.75$ & 0.97 \\
\hline Serum creatinine & $1.17 \pm 1.02$ & $0.95 \pm 0.39$ & 0.016 \\
\hline Femoral route & 12 & 9 & \\
\hline Radial route & 159 & 87 & \\
\hline Total no. of lesions treated & 247 & 130 & \\
\hline Multi & 24 & 9 & \\
\hline Double & 120 & 60 & \\
\hline Single & 103 & 61 & \\
\hline
\end{tabular}

Abbreviations: ACS, acute coronary syndrome; CSA, chronic stable angina; DM, diabetes mellitus; HTN, hypertension; LV, left ventricular. 
Hence, CAD with acute severe presentation and complications in the form of LV and renal dysfunctions were more common in female patients who had type II DM than those who did not.

When primary outcomes in the form of MACE were considered up to 1 year post intervention in group A, three cases of deaths, one case of acute stent occlusion, one subacute stent occlusion, and one peri-procedural MI were observed, whereas in group B one death was observed, and this difference was statistically significant.

Among the 171 patients with type II diabetes, at the time of presentation 103 (60.3\%) patients had random blood sugar (RBS) of more than $200 \mathrm{mg} / \mathrm{dL}$ and 68 (39.7\%) had RBS of less than $200 \mathrm{mg} / \mathrm{dL}$. In addition, all the observed MACE were seen in patients with RBS more than $200 \mathrm{mg} / \mathrm{dL}$ with statistical significance $(p<0.05)$ and the mean RBS in patients who had succumbed to MACE was $268 \mathrm{mg} / \mathrm{dL}$.

When secondary outcomes in the form of CSA, statin-induced myopathy, rhabdomyolysis, and procedural complications were compared, one case of CSA, one statin-induced myopathy, and one rhabdomyolysis were observed in group $\mathrm{B}$, whereas one case of CSA, one CIN, one chronic thrombus right radial artery, and one pseudoaneurysm and rupture were observed in group A ( - Table 2, - Fig. 3).

\section{Discussion}

Women were thought to have a lower risk of developing CAD traditionally, but due to increased incidence of coronary risk factors like HTN and DM, the risk of CAD has increased significantly in women. Despite this, CAD remains as an underrecognized condition in women. Limited data exist on the frequency of $\mathrm{MI}$ in female patients. The incidence of an MI over a 10-year follow-up was 5.2/1,000 in women aged 35 to 44 years as reported in Framingham heart study. ${ }^{18}$ It was eight to nine times greater in women aged 55 to 64 years.

In our study, the incidence of MI in women between 35 and 44 years and between 55 and 64 years was 4.2 and $12.4 \%$, respectively, which is similar to the previous studies. Incidence of $\mathrm{CAD}$ has increased with age, but younger age of presentation is more common in patients with type II diabetes. HTN also being an important risk factor for development and progression of CAD is present in most patients with diabetes at the time of diagnosis. In our study, $86 \%$ of the patients with diabetes were hypertensive but among nondiabetics only $53 \%$ were hypertensive. The mean of mean arterial blood pressure (MABP) in both the groups were 110 and $98 \mathrm{~mm} \mathrm{Hg}$ with higher levels seen in those with diabetes. Incidence of MACE in patients with type II diabetes after PCI for CAD had direct correlation with sugar control according to our study. Our study findings also support the existing knowledge that the extent of the disease in the coronary arteries is greater among patients with type II DM.

The Thrombolysis and Angioplasty in Myocardial Infarction (TAMI) trial showed that compared with the nondiabetics, patients with type II DM had a significantly higher incidence of multi-vessel disease. In our study also, the incidence of multi-vessel disease was greater in patients with type II diabetes.

In patients with diabetes, at the time of presentation, the mean creatinine was higher(statistically significant) compared with the nondiabetic patients, which can be explained by increased prevalence of chronic kidney disease in diabetics. Also, patients with diabetes are more prone for acute kidney injury because of less kidney reserve

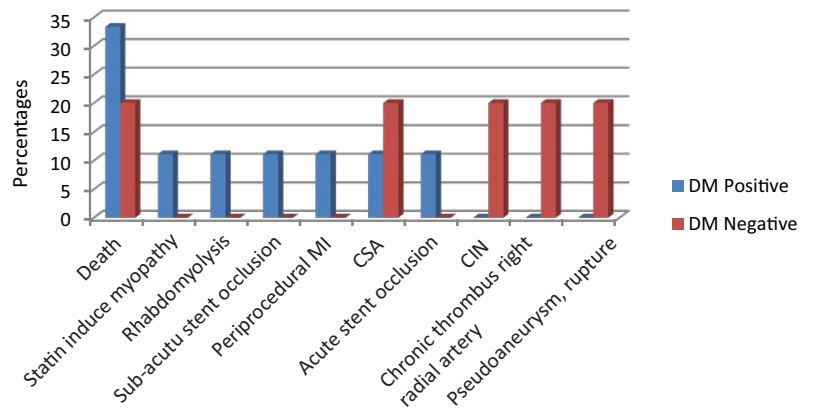

Fig. 3 Comparison of $\mathrm{PCl}$ outcomes in diabetics versus nondiabetics. CIN, contrast-induced nephropathy; CSA, chronic stable angina; DM, diabetes mellitus; MI, myocardial infarction.

Table 2 Comparison of $\mathrm{PCl}$ outcomes in type II diabetes mellitus versus nondiabetics

\begin{tabular}{|l|l|l|}
\hline Outcomes & DM positive & DM negative \\
\hline Death & $3(33.33 \%)$ & $1(20 \%)$ \\
\hline Statin-induce myopathy & $1(11.11 \%)$ & 0 \\
\hline Rhabdomyolysis & $1(11.11 \%)$ & 0 \\
\hline Subacute stent occlusion & $1(11.11 \%)$ & 0 \\
\hline Periprocedural MI & $1(11.11 \%)$ & 0 \\
\hline CSA & $1(11.11 \%)$ & $1(20 \%)$ \\
\hline Acute stent occlusion & $1(11.11 \%)$ & 0 \\
\hline CIN & 0 & $1(20 \%)$ \\
\hline Chronic thrombus right radial artery & 0 & $1(20 \%)$ \\
\hline Pseudo aneurysm, rupture & 0 & $1(20 \%)$ \\
\hline
\end{tabular}

Abbreviations: CIN, contrast-induced nephropathy; CSA, chronic stable angina; DM, diabetes mellitus; MI, myocardial infarction; PCl, percutaneous coronary intervention. 
function. LV dysfunction at the time of presentation of CAD was observed in greater numbers in patients with type II DM though the difference was not statistically significant.

Postprocedural complications in the form of stent thrombosis and periprocedural MI were more common in female patients with diabetes compared with nondiabetic female patients, reinforcing the fact that type II DM has negative impact on prognosis after $\mathrm{PCI}$ and also poorly controlled type II DM has strong association with MACE. The possible explanations include increased platelet activity that is seen in patients with poorly controlled DM. ${ }^{17,19}$

Treatment-related complications like statin-induced myopathy and rhabdomyolysis were more common in female patients with diabetes compared with those without type II DM. ${ }^{18}$

\section{Conclusion}

Our study suggests that in female CAD patients, type II DM has strong impact on the severity of presentation. Complications such as LV and renal dysfunctions as well as the multi-vessel involvement are more common in patients with type II diabetes. After PCI, patients with type II diabetes have experienced more MACE, statin-induced myopathy, and rhabdomyolysis compared with nondiabetic patients. To conclude, in female CAD patients, diabetes is a poor prognostic marker in the form of more severe presentation and poorer outcomes after treatment.

\section{Conflict of Interest}

None.

\section{References}

1 Danaei G, Finucane MM, Lu Y, et al; Global Burden of Metabolic Risk Factors of Chronic Diseases Collaborating Group (Blood Glucose). National, regional, and global trends in fasting plasma glucose and diabetes prevalence since 1980: systematic analysis of health examination surveys and epidemiological studies with 370 country-years and 2.7 million participants. Lancet 2011;378(9785):31-40

2 Eaker ED, Chesebro JH, Sacks FM, Wenger NK, Whisnant JP, Winston M. Cardiovascular disease in women. Circulation 1993;88(4 Pt 1):1999-2009

3 Mosca L, Mochari H, Christian A, et al. National study of women's awareness, preventive action, and barriers to cardiovascular health. Circulation 2006;113(4):525-534

4 GBD 2013 Mortality and Causes of Death Collaborators. Global, regional, and national age-sex specific all-cause and cause-specific mortality for 240 causes of death, 1990-2013: a systematic analysis for the Global Burden of Disease Study 2013. Lancet 2015;385(9963):117-171

5 Sullivan AK, Holdright DR, Wright CA, Sparrow JL, Cunningham D, Fox KM. Chest pain in women: clinical, investigative, and prognostic features. BMJ 1994;308(6933):883-886
6 Rich-Edwards JW, Manson JE, Hennekens $\mathrm{CH}$, Buring JE. The primary prevention of coronary heart disease in women. $\mathrm{N}$ Engl J Med 1995;332(26):1758-1766

7 Zuanetti G, Latini R, Maggioni AP, Santoro L, Franzosi MG. Influence of diabetes on mortality in acute myocardial infarction: data from the GISSI-2 study. J Am Coll Cardiol 1993;22(7):1788-1794

8 Huxley R, Barzi F, Woodward M. Excess risk of fatal coronary heart disease associated with diabetes in men and women: meta-analysis of 37 prospective cohort studies. BMJ 2006;332(7533):73-78

9 Hu G, Jousilahti P, Qiao Q, Peltonen M, Katoh S, Tuomilehto J. The gender-specific impact of diabetes and myocardial infarction at baseline and during follow-up on mortality from all causes and coronary heart disease. J Am Coll Cardiol 2005;45(9):1413-1418

10 Lee PY, Alexander KP, Hammill BG, Pasquali SK, Peterson ED. Representation of elderly persons and women in published randomized trials of acute coronary syndromes. JAMA 2001;286(6):708-713

11 Kim ESH, Carrigan TP, Menon V. Enrollment of women in National Heart, Lung, and Blood Institute-funded cardiovascular randomized controlled trials fails to meet current federal mandates for inclusion. J Am Coll Cardiol 2008;52(8):672-673

12 Lansky AJ, Hochman JS, Ward PA, et al; American College of Cardiology Foundation; American Heart Association. Percutaneous coronary intervention and adjunctive pharmacotherapy in women: a statement for healthcare professionals from the American Heart Association. Circulation 2005;111(7):940-953

13 Blomkalns AL, Chen AY, Hochman JS, et al; CRUSADE Investigators. Gender disparities in the diagnosis and treatment of nonST-segment elevation acute coronary syndromes: large-scale observations from the CRUSADE (Can Rapid Risk Stratification of Unstable Angina Patients Suppress Adverse Outcomes With Early Implementation of the American College of Cardiology/American Heart Association Guidelines) National Quality Improvement Initiative. J Am Coll Cardiol 2005;45(6):832-837

14 White HD, Barbash GI, Modan M, et al. After correcting for worse baseline characteristics, women treated with thrombolytic therapy for acute myocardial infarction have the same mortality and morbidity as men except for a higher incidence of hemorrhagic stroke. The Investigators of the International Tissue Plasminogen Activator/Streptokinase Mortality Study. Circulation 1993;88(5 Pt 1):2097-2103

15 Hochman JS, Tamis JE, Thompson TD, et al. Sex, clinical presentation, and outcome in patients with acute coronary syndromes. Global Use of Strategies to Open Occluded Coronary Arteries in Acute Coronary Syndromes IIb Investigators. N Engl J Med 1999;341(4):226-232

16 Mehilli J, Ndrepepa G, Kastrati A, et al. Gender and myocardial salvage after reperfusion treatment in acute myocardial infarction. J Am Coll Cardiol 2005;45(6):828-831

17 Davi G, Catalan I, Averna M. Thromboxane biosynthesis and platelet function in type II diabetes mellitus. N Engl J Med 1990;322(25):1769-1774

18 Bitzur R, Cohen H, Kamari Y, Harats D. Intolerance to statins: mechanisms and management. Diabetes Care 2013;36(Suppl 2): S325-S330

19 Davì G, Patrono C. Platelet activation and atherothrombosis. N Engl J Med 2007;357(24):2482-2494 